\title{
ANÁLISE MULTICRITÉRIO EM DADOS SOBRE EMPREENDEDORISMO: UM ESTUDO BIBLIOMÉTRICO
}

\section{MULTICRITERIA ANALYSIS ON ENTREPRENEURSHIP DATA: A BIBLIOMETRIC STUDY}

\author{
Marta Duarte de Barros* E-mail: marta uff@hotmail.com \\ Helder Gomes Costa* E-mail: helder.hgc@gmail.com \\ Glauco Barbosa da Silva*E-mail: glaucos@id.uff.br \\ Mara Regina dos Santos Barcelos* E-mail: marabarceloss@gmail.com \\ Altina Silva Oliveira* E-mail: altinaadm@gmail.com \\ *Universidade Federal Fluminense (UFF), Niterói, RJ
}

Resumo: Devido à sua importância no estabelecimento de empresas e na formação de pessoas com características empreendedoras, o empreendedorismo é um tema acadêmico amplamente discutido, contribuindo para o crescimento econômico em termos nacionais e internacionais. O presente artigo tem como objetivo apresentar uma compilação de informações sobre a utilização da análise multicritério para tratamento de dados relacionados ao empreendedorismo. Uma pesquisa bibliométrica foi realizada nas bases Scopus e ISI Web of Science, acessadas a partir do Portal de Periódicos da Capes, em março de 2016. Um total de 37 artigos foram retornados/ revelados, excluídos os duplicados e os que não estavam de acordo com objetivo da pesquisa, 13 foram selecionados para análise, resultando nos seguintes aspectos de interesse da amostra: ciclo de produção; palavras-chave; país de origem; principais autores e periódicos; áreas do conhecimento relacionadas. Como contribuição, espera-se a construção de uma visão global da literatura existente sobre multicritério na análise de dados de empreendedorismo.

Palavras-chave: Multicritério. Empreendedorismo. Bibliometria. Scopus. ISI Web of Science.

Abstract: Due to its importance in the establishment of businesses and in the training of people with entrepreneurial characteristics, entrepreneurship is a widely discussed academic subject, contributing to economic growth in both national and international terms. This article's objective is to present a compilation of information about the use of multicriteria analysis for the treatment of data on entrepreneurship. A bibliometric survey was performed in the Scopus and ISI Web of Science databases, accessed from the Capes journal portal in March 2016. A total of 37 articles were found. After excluding duplicates and those articles outside the scope of the research objective, 13 were selected for analysis, resulting in the following aspects of interest of the sample: production cycle; keywords; country of origin; main authors and journals; related fields of knowledge. As a contribution, this article is expected to build an overview of the existing literature on multicriteria in the data analysis of entrepreneurship.

Keywords: Multicriteria. Entrepreneurship. Bibliometry. Scopus. ISI Web of Science.

\section{INTRODUÇÃO}

Contribuindo para o crescimento econômico dos países em termos nacionais e internacionais; e devido à sua grande importância tanto no estabelecimento de 
empresas, quanto na formação de pessoas com características empreendedoras, um dos assuntos acadêmicos mais discutidos atualmente é o empreendedorismo.

Segundo Stamboulis e Barlas (2014), empreendedorismo e novas empresas tornam-se um potencial de valor para o desenvolvimento e crescimento econômico na sociedade moderna. Dessa forma, é uma demanda para as universidades cultivar a capacidade empreendedora dos alunos para se adaptar à transformação econômica e modernização, bem como na construção e desenvolvimento da economia empresarial. Também é importante para melhorar o sistema de ensino em faculdades e universidades, o reforço da formação de inovação do talento empresarial (YANG; ZHAO, 2014). Nas últimas décadas, uma prática comum nas universidades tem sido a inclusão do empreendedorismo na grade dos cursos de engenharia (EVANS et al.,2007). Segundo Saee (1996), ensinar características empreendedoras para os alunos é uma maneira de possibilitar o alcance do sucesso.

Ruda et al. (2012) sugerem que as oportunidades inovadoras a nível regional, nacional e internacional são capazes de construir condições básicas para alcançar a estabilidade econômica. Para lidar com tais questões inovadoras, uma proposta é a disseminação da cultura empreendedora em projetos educacionais em cursos de graduação. Na engenharia de produção, por exemplo, a disciplina empreendedorismo tem sido implementada. Estudos dos fatores que influenciam o desempenho do negócio e a relação com o empreendedorismo e a inovação têm atraído o interesse de um grande número de pesquisadores (GONÇALVES FILHO et al., 2012).

Os países avançados, como Estados Unidos e Japão, têm promovido fortemente a educação empreendedora, fornecendo medidas de apoio relevantes para incentivar os formandos como, por exemplo, abrir uma empresa para aumentar a empregabilidade dos mesmos (CHOU et al., 2010).

Duval-Couetil et al. (2014) afirmam que o envolvimento de alunos de graduação em proteção à propriedade intelectual leva a interessantes questões relacionadas com a melhor forma de alinhar os interesses dos estudantes com as políticas e práticas institucionais.

Segundo um estudo realizado por Galloway et al. (2006) no Reino Unido, o empreendedorismo tem um efeito positivo para os estudantes de engenharia, além 
de aumentar os níveis de inovação nas empresas, contribuindo assim para aumentar a competitividade.

$\mathrm{Na}$ última década, o número de programas de empreendedorismo nas universidades destinados aos estudantes de engenharia tem crescido substancialmente. No entanto, poucos estudos têm analisado as práticas e crenças dos instrutores nesses programas. Assim, Zappe et al. (2013) afirmam que entender essas práticas e crenças contribuirá para 0 desenvolvimento de modelos pedagógicos e teóricos para conduzir educação para o empreendedorismo.

Além disso, as barreiras, tanto comerciais como culturais, estão sendo eliminadas, incorporando inovações nas relações de trabalho e empregabilidade, favorecendo assim os novos meios de riqueza para a sociedade e o sucesso organizacional (WILLERDING; PRADO; LAPOLLI, 2012).

Trabalhos anteriores sobre o tema como Silva et al. (2015a) consideram que a descoberta de oportunidades empresariais constitui uma habilidade empreendedora importante, uma fonte de vantagem competitiva, e uma importante área de conteúdo em educação para o empreendedorismo em engenharia.

Silva, Furtado e Zanini (2015) realizaram um estudo no qual se observa que, na maioria dos empreendedores, há tendência à estabilidade nos negócios, e ainda, afirmam que o crescimento e contribuição das mulheres na economia brasileira nos últimos anos. Entretanto, a elevada burocracia e carga tributária do país são fatores desestimulantes.

Devido à importância do Empreendedorismo, não só para os estudantes, mas também pela possibilidade de contribuir para o crescimento econômico, novas maneiras de avaliar as percepções e tratar dados relativos ao tema estão sendo testados, como por exemplo, a utilização de técnicas multicritério como MCDA (Multicriteria Decision Analysis) e MCDM (Multicriteria Decision Making).

Diante deste cenário, surge o seguinte questionamento: Quais são os autores, ciclos de produção bibliográfica, instituições, áreas, periódicos e países com maior número de publicações referentes à aplicação da análise multicritério ao tema empreendedorismo?

O objetivo deste artigo é apresentar uma compilação das informações sobre a utilização da análise multicritério para tratamento dos dados relacionados ao empreendedorismo, fornecendo diferentes tipos de análises comparativas. Deste modo, 
espera-se como contribuição deste trabalho estabelecer um referencial bibliográfico, formando um núcleo inicial, que possibilite uma visão global da aplicação da análise multicritério para analisar dados relativos ao empreendedorismo.

O trabalho está organizado como a seguir: na seção 2, um breve resumo sobre análise multicritério é apresentado; a seção 3 trata do uso da bibliometria; na seção 4 são descritos conceitos de empreendedorismo; o método de pesquisa aplicado nesta revisão é descrito na seção 5; na seção 6 são apresentados os resultados obtidos na pesquisa bibliométrica; encerrando-se com uma breve conclusão.

\section{ANÁLISE MULTICRITÉRIO}

Há uma grande variedade de métodos de análise multicritério, que se diferem entre si através da maneira que os critérios são tratados. Segundo Guglielmetti, Marins e Salomon (2003) os seguintes fatores são considerados:

a) Modo pelo qual os critérios são julgados;

b) Obtenção de pesos dos critérios;

c) Tratamento dos pesos dos critérios.

Guglielmetti, Marins e Salomon (2003) fizeram uma comparação entre três desses métodos: MAHP, AHP e ELECTRE I, no que diz respeito à entrada de dados, conforme exposto no Quadro 1.

Quadro 1 - Comparação entre métodos de análise multicritério

\begin{tabular}{|l|c|c|c|}
\hline \multicolumn{1}{|c|}{ ENTRADA DE DADOS (INPUT) } \\
\hline \multirow{2}{*}{ DESCRIÇÃo } & AHP & MAHP & ELECTRE I \\
\cline { 2 - 4 } & Sim & Sim & Não \\
\hline Utilização em decisões com vários níveis. & Sim & Não & Não \\
\hline Restrições quanto à quantidade de elementos em um nível. & Alta & Média/Alta & Baixa \\
\hline $\begin{array}{l}\text { Quantidade de julgamentos em problemas com muitos critérios e } \\
\text { alternativas. }\end{array}$ & Não & Sim & Sim \\
\hline Necessidade de processar os dados antes de usar. & Sim & Sim & Sim \\
\hline Possibilidade de tratar dados quantitativos e qualitativos. & Sim & Sim & Sim \\
\hline Possibilidade de lidar com problemas do tipo técnico. & Não & Não & Não \\
\hline Possibilidade de tratar critérios/alternativas dependentes. & Não & Sim & Não \\
\hline Possibilidade de criar escalas de julgamento no contexto. & & \\
\hline
\end{tabular}

Fonte: Adaptado de Guglielmetti, Marins e Salomon (2003)

\section{USO DA BIBLIOMETRIA}

O uso da bibliometria é cada vez mais frequente entre os pesquisadores, pois 
fornece uma ampla visão do tema de interesse, independente dos objetivos. Sendo assim, nesta seção são apresentados alguns trabalhos que utilizaram a bibliometria com objetivos diferentes.

Silva, Costa e Barros (2015b) apresentaram uma revisão sobre Educação Empreendedora em cursos de engenharia, através de um estudo bibliométrico. A princípio 16.835 documentos foram encontrados, e após a aplicação de filtros e restrição a artigos publicados em revistas, e no campo de engenharia, 74 artigos foram selecionados e mapeados por um modelo de literatura.

Valle, Gutierrez e Costa (2013) identificaram vários indicadores que permitiram mapear a produção científica em Gestão de Processos, tanto genérica como na área da Saúde, através do uso da bibliometria. Além disso, identificaram alguns gaps, sendo o principal deles, entre a produção genérica e na Saúde, sendo que apenas $6 \%$ da produção indexada pela base Scopus em Gestão de Processos está voltada para a Saúde.

Zyoud et al. (2014) tiveram como objetivo analisar a produção de pesquisa de 13 países Árabes em periódicos de toxicologia, através de estudo bibliométrico. Foram encontrados 1240 artigos pulverizados em 73 periódicos.

Zhang, Xie e Ho (2009) utilizaram o estudo bibliométrico para avaliar quantitativamente as tendências de pesquisa abstrata em compostos orgânicos voláteis, usando a base de dados SCI (Scientific Citation Index) no período de 1992 a 2007. Foram encontradas 11.048 publicações que preenchiam os critérios de seleção.

Tian, Wen e Hong (2008) realizaram uma análise bibliométrica para avaliar a produção científica global de artigos do Sistema de Informação Geográfica (GIS), no período de 1997 a 2006 em qualquer revista de todas as categorias de assunto do Science Citation Index compilado pelo Institute for Scientific Information (ISI) EUA. Os resultados mostram que 9.849 artigos foram publicados, pulverizados em 1.918 periódicos, e listadas em 202 categorias de assuntos estabelecidas pelo ISI.

Van-Raan (2006) utilizou o estudo bibliométrico para verificar a correlação estatística entre o índice de Hirsch (h-) e vários indicadores, através dos resultados de um estudo com 147 grupos de pesquisa universitários nos Países Baixos, com um total de 700 pesquisadores. 


\section{EMPREENDEDORISMO}

O Empreendedorismo é definido por Karimi et al. (2010) como o comportamento de uma pessoa que mostra os traços: necessidade de realização, necessidade de poder e tendência a assunção de riscos, e competitividade necessários para obter ou alcançar alguma coisa na vida, para pesquisar e se tornar autossuficiente.

Gomezelj e Kušce (2013) definem Empreendedorismo como um processo global, com tendência de se tornar mais importante nos próximos anos. CristianAurelian e Cristina (2012) consideram o empreendedorismo como um processo inovador através do qual os empresários identificam as oportunidades de negócios para explorá-las através da atribuição de recursos e criação de valor.

O Empreendedorismo é relevante para todos os setores, tecnológicos ou tradicionais, para pequenas e grandes empresas, além de contribuir para a criação de emprego e crescimento econômico; e tem sido cada vez mais reconhecido como um dos principais motores do desenvolvimento econômico ((CRISTIAN-AURELIAN; CRISTINA, 2012); (QIAN; HAYNES, 2014)).

Tsai et al. (2014) concordam que uma política empreendedora é projetada para abordar as áreas de motivação, oportunidades e habilidades, e que com políticas empreendedoras adequadas, mais pessoas serão encorajadas a iniciar um negócio, que impulsionará a economia.

O objetivo da política de espírito empreendedor é criar um ambiente e um sistema de apoio que favoreça o surgimento de novos empreendedores, bem como o crescimento de novas empresas (TSAI; KUO, 2011).

\subsection{Empreendedorismo na Educação}

Pardede e Lyons (2012) afirmam que o empreendedorismo na educação consiste em ensinar aos alunos de graduação de tecnologia da informação, o processo, o conhecimento e as habilidades necessárias para começar um novo negócio. Enquanto outros cursos do programa de TI focam em habilidades para resolver problemas.

Empreendedorismo na educação tenta preparar as pessoas, especialmente os jovens, para serem responsáveis, assumirem riscos, gerirem os negócios e 
aprenderem com os resultados por imersão em experiências de aprendizagem da vida real (KARIMI et al., 2010).

De acordo com Ruda et al. (2009), o objetivo do empreendedorismo na educação não deve ser apenas a formação individual, mas também, as competências empresariais gerais no âmbito de um cruzamento interdisciplinar. Esta abordagem ao ensino de base empresarial em vigor incentiva surgimento de novas ideias de negócio dos estudantes.

Se a universidade cultivar ativamente a capacidade empreendedora de seus alunos, eles melhorarão o sistema de gestão, e o empreendedorismo para educação criará base de formação para o empreendedorismo e assim por diante. A universidade empreendedora responde, assim, através da geração de transferência de tecnologia, a criação de empresas baseadas no conhecimento e no capital humano (YANG; ZHAO, 2014).

O empreendedorismo surgiu como um mecanismo importante para a geração de retornos sociais em termos de crescimento econômico e criação de emprego, mas, empreendedorismo na educação ainda é algo recente na Europa e no debate sobre a necessidade e a forma de introdução de cursos de empreendedorismo específicos no ensino superior (PAPAYANNAKIS et al., 2008).

Empreendedorismo na educação tem um papel fundamental no desenvolvimento do empreendedorismo como processo dinâmico de visão, mudança e criação de educação, e fornece energia e paixão para a criação e implementação de novas ideias em uma base contínua. Instituições de engenharia, na sua maioria, desempenham um papel passivo, resultando em muitos mitos e receios entre os estudantes sobre empreendedorismo (KHANDUJA; KAUSHIK, 2009).

\subsection{Empreendedorismo \& Multicritério}

Os 13 artigos selecionados foram analisados com o foco na utilização de análise multicritério em dados sobre empreendedorismo.

O estudo apresentado por Ferreira et al. (2015) afirma que a Orientação Empreendedora Individual vai além de identificar traços, atitudes, contextos ou intenções, mas, também compreender como estes elementos interagem entre si. Sendo assim, desenvolveram um framework, integrando o uso de mapas cognitivos com o método Macbeth, com o objetivo de auxiliar na medição da Orientação Empreende- 
dora Individual. Em sua conclusão, os autores salientam para o cuidado com a subjetividade do estudo.

Gomes, Machado e Rangel (2015) utilizaram a integral de Choquet (que utiliza o método Fuzzy) para resolver problemas de múltiplas escolhas, quando há interações entre os critérios de decisão. Foram definidos nove critérios de avaliação. Os dados foram cedidos pelo SEBRAE-RJ, contendo 11 projetos. Os autores concluem que o método utilizado é eficaz a proposta do trabalho.

Deluyi et al. (2014) afirmam que o gerenciamento da cadeia de suprimentos (SCM) facilita a criação de vantagem competitiva sustentável que pode causar novos empreendimentos e desenvolvimento de crescimento de negócios. Sendo assim, utilizaram MCDM na seleção de fornecedores e análise envoltória de dados (DEA) para verificar a eficiência dos mesmos. O objetivo foi identificar potenciais empreendedores e critérios de desempenho.

Rostamzadeh, Ismall e Noubar (2014) fizeram um estudo para avaliar e priorizar a intensidade empreendedora de pequenas e médias empresas na Malásia, usando técnicas de decisão multicritério (MCDM). As técnicas VIKOR e TOPSIS também foram utilizadas neste estudo. Trinta empresas do setor de manufatura foram investigadas neste estudo, os autores sugerem que a pesquisa seja aplicada em outros países, em setores semelhantes, além do uso de outros métodos multicritério, como ELECTRE, PROMETHEE e DEMATEL.

Tsai et al. (2014) questionam quanto esforço deve um governo investir em melhorias políticas, a fim de alcançar um nível aceitável de crescimento econômico. Para os autores esta é uma questão crítica que precisa de um modelo de avaliação com uma metodologia adequada, visto que estudos para avaliar sistematicamente os vários elementos conflitantes de uma política de empreendedorismo ainda são incipientes. Sendo assim, propuseram um modelo que integra o processo de rede analítico (ANP) e a técnica VIKOR para avaliar as políticas de empreendedorismo e, em seguida priorizar as ações de melhoria. $O$ estudo de caso foi realizado em Taiwan. Os autores salientam que o uso do modelo proposto fornece apenas recomendações para a construção de uma política ideal.

Rezaei, Ortt e Scholten (2013) realizaram um estudo para mensurar a Orientação ao Empreendedorismo (EO) das empresas. Para isso utilizaram o método multicritério AHP. Foram selecionadas 59 pequenas e médias empresas na Holanda, 
totalizando 131 entrevistados. Os resultados mostraram que a pró-atividade é o critério mais importante, seguido pela capacidade de inovação. Além disso, existem diferenças consideráveis quando se trata dos pesos dos itens.

Buracas, Zvirblis e Joksiene (2012) se propuseram a mensurar as vantagens do macro empreendedorismo, através da abordagem de competitividade econômica dos países da Europa. Eles afirmam que a competitividade econômica pode ser interpretada como um fenômeno multidimensional e com isso, pode ser analisada através de multicritério. A técnica proposta permitiu que critérios múltiplos da competitividade econômica fossem avaliados e orientados para as prioridades estratégicas nacionais, com base nos pilares de fatores adequados.

Han et al. (2012) desenvolveram um modelo de avaliação do ambiente empreendedor baseado na teoria fuzzy. O modelo foi utilizado em onze regiões da província de Hebei, e fornece uma ferramenta precisa, efetiva e sistemática de apoio à decisão.

Jamali et al. (2012) fizeram uma revisão sobre estudos existentes sobre conhecimento empresarial para identificar suas limitações, do ponto de vista feminino. Um questionário foi aplicado para 438 mulheres, funcionárias do setor cooperativo do Irã, além de 40 horas de entrevistas com empresárias do setor. A técnica de multicritério AHP foi utilizada nos dados para determinar o significado de cada uma das barreiras, mostrando que as barreiras de infraestrutura têm impacto máximo, enquanto as barreiras culturais têm um impacto mínimo.

Tsai et al. (2011) propuseram um modelo integrado, que traz uma combinação entre o Processo de Decisões e Laboratório de Avaliação (DEMATEL), o processo de rede analítica (ANP) e métodos de programação de objetivos zero-um (ZOGP). A relação interdependente entre critérios e alternativas de avaliação de políticas complexas foi considerada, e as restrições dos orçamentos foram abordadas. O estudo de caso foi realizado em pequenas e médias empresas em Taiwan. Os resultados da avaliação mostram que três alternativas (centro de incubação, assistência financeira e plataformas de compartilhamento de conhecimento) são selecionadas sob restrições orçamentárias. Os resultados indicam que oferecendo serviços e informações de consultoria, além de fornecer apoio financeiro e criar um site de banco de dados de conhecimento, os governos podem aumentar a taxa de sucesso 
das empresas, incentivando os indivíduos a iniciarem PME, facilitando o crescimento econômico.

Schwartz e Gothner (2009) afirmam que recursos públicos são dedicados ao estabelecimento e funcionamento de incubadoras de empresas (Bls), que são vistas como catalisadoras para a promoção do empreendedorismo. Os autores dizem que grande parte das pesquisas que se concentra na eficácia das iniciativas de incubadoras, e em como medir o desempenho da incubadora, e mesmo assim, ainda há uma falta de compreensão de como determinar quais incubadoras são mais eficazes. Sendo assim, esse foi o foco de seu trabalho, e com base nos dados de 410 empresas, compararam a eficácia a longo prazo de cinco IEs na Alemanha, aplicando a técnica de classificação de preferências "PROMETHEE".

Chen et al. (2006) forneceram uma abordagem quantitativa de tomada de decisão multicritério para a gestão do conhecimento na educação empresarial através de um processo de rede de conhecimento analítico (KANP). Utilizaram a abordagem KANP, que integra uma classificação industrial padrão com o processo de rede analítica (ANP). O CEEM foi construído para aplicar o método KANP na avaliação de casos de ensino. Os resultados mostram que há oito clusters e 178 nós no modelo KANP. O CEEM, e pesquisas experimentais sobre a avaliação de casos de ensino revelam que o método KANP é eficaz na condução de conhecimento para a educação em empreendedorismo.

Sha, Hung e Lin (2004) realizaram uma análise que emprega uma estrutura Fuzzy (FMCDM) com uma fórmula empírica para examinar a sua praticabilidade e utilidade. Com base em questões empresariais, técnicas e ambientais, sete estratégias foram definidas. A análise foi realizada em Taiwan, e os resultados mostram que três clusters são extraídos entre as sete estratégias pela abordagem de C-Meios Fuzzy.

\section{METODOLOGIA}

As bases de dados ISI Web of Science e Scopus foram utilizadas para realizar a pesquisa bibliométrica, acessadas a partir do Portal Capes em março de 2016.

O trabalho bibliométrico em questão é descritivo e exploratório com abordagem quantitativa, a partir do uso de ferramentas da Estatística Descritiva.

A pesquisa foi estruturada da seguinte forma: 
1. Revisão bibliográfica: Utilizou-se do Portal da Capes para realizar a pesquisa, algumas das referências selecionadas foram utilizadas para a escrita da revisão da literatura.

2. Levantamento Bibliométrico: Na base de dados Scopus utilizou-se da pesquisa avançada. A seguinte estrutura foi utilizada: TITLE-ABS-KEY (multicriteria OR "multiplecriteria" OR "mcdm" OR "mcda") AND TITLE-ABS-KEY ("entrepreneurship") por articletitle, abstract e keywords, resultando em 18 artigos.

$\mathrm{Na}$ base de dados ISI Web of Science também se utilizou da pesquisa avançada e a seguinte estrutura foi utilizada: (("multicriteria" $O R$ "multiplecriteria" $O R$ "mcdm" OR "mcda") AND ("entrepreneurship")) por articletitle, abstract e keywords, resultando em 19 artigos.

O objetivo do levantamento bibliométrico foi fazer uma análise dos artigos que abordam o tema "empreendedorismo e análise multicritério", a partir dos seguintes tópicos:

a) Quantitativo de artigos por ano;

b) Análise das palavras-chave;

c) Análise do país de origem;

d) Principais Autores no tema;

e) Áreas do conhecimento em destaque; e

f) Principais Periódicos no tema.

Esses tópicos foram definidos com base no trabalho proposto por Costa (2010), que propôs um modelo (webibliomining) para mineração de fontes bibliográficas baseado em ferramentas de acesso e busca de dados na internet.

\section{RESULTADOS}

A pesquisa bibliométrica realizada nas bases Scopus e ISI Web of Science considerou a expressão "multicriteria" OR "multiplecriteria" OR "mcdm" OR "mcda" $e$ "entrepreneurship" por articletitle, abstract e keywords, com as respectivas peculiaridades de pesquisa como mostrado na metodologia.

Após realizar as pesquisas, foi efetuada uma análise para identificar artigos duplicados nas diferentes bases. Como resultado, identificou-se que nove dos artigos encontrados na Scopus também foram encontrados na ISI Web of Science. Assim, desconsiderando os duplicados, foram analisados 19 artigos. 
Dando continuidade ao trabalho, uma leitura dos resumos destes 19 artigos foi realizada, após o descarte dos artigos que não se enquadravam no tema pesquisado, 13 artigos foram escolhidos para o atual estudo, conforme apresentado no Quadro 2, com os autores, títulos, ano e periódico.

Quadro 2 - Artigos que compõem a base de referência

\begin{tabular}{|c|c|c|c|}
\hline Autores & Título & Ano & Periódico \\
\hline $\begin{array}{l}\text { Buracas, A.; Zvirblis, A.; } \\
\text { Joksiene, L. }\end{array}$ & $\begin{array}{l}\text { Measurement of entrepreneurship } \\
\text { macro surrouding advantages: } \\
\text { Coutry's economic competitiveness } \\
\text { approach. }\end{array}$ & 2012 & Engineering Economics \\
\hline $\begin{array}{l}\text { Chen, Z.; Li, H.; Kong, S. } \\
\text { C.; Xu, Q. }\end{array}$ & $\begin{array}{l}\text { An analytic knowledge network pro- } \\
\text { cess for construction entrepreneurship } \\
\text { education. }\end{array}$ & 2006 & $\begin{array}{l}\text { Journal of Management } \\
\text { Development }\end{array}$ \\
\hline $\begin{array}{l}\text { Deluyi, A. H. M.; Sofian, } \\
\text { S.; Hamid, A. B. A.; } \\
\text { Kazemi, M. }\end{array}$ & $\begin{array}{l}\text { Screening efficiency and entrepre- } \\
\text { neurship potential in automotive sup- } \\
\text { phers using DEA. }\end{array}$ & 2014 & $\begin{array}{l}\text { Applied Mechanics \& } \\
\text { Materials }\end{array}$ \\
\hline $\begin{array}{l}\text { Ferreira, F. A.; Marques, } \\
\text { C. S.; Bento, P.; Ferreira, } \\
\text { J. J.; Jalali, M. S. }\end{array}$ & $\begin{array}{l}\text { Operationalizing and measuring indi- } \\
\text { vidual entrepreneurial orientation using } \\
\text { cognitive mapping and MCDA tech- } \\
\text { iniques. }\end{array}$ & 2015 & $\begin{array}{l}\text { Journal of Business Re- } \\
\text { search }\end{array}$ \\
\hline $\begin{array}{l}\text { Gomes, L. F. A.; Macha- } \\
\text { do, M. A. S.; Rangel, L. } \\
\text { A. D. }\end{array}$ & $\begin{array}{l}\text { The multiple choice problem with inter- } \\
\text { actions between criteria. }\end{array}$ & 2015 & Pesquisa Operacional \\
\hline $\begin{array}{l}\text { Han, Jing-Yuan; Yang, } \\
\text { Yan-Bo; Zhao, Yun-He. }\end{array}$ & $\begin{array}{l}\text { Evaluation of entrepreneurial environ- } \\
\text { ment based on fuzzy comprehensive } \\
\text { evaluation method. }\end{array}$ & 2012 & $\begin{array}{l}\text { Machine Learning and } \\
\text { Cybernetics (ICMLC). } \\
2012 \text { International Confe- } \\
\text { rence on IEEE. }\end{array}$ \\
\hline $\begin{array}{l}\text { Jamali, R.; Jahantigh, F. } \\
\text { F.; Sazvar, A.; Maleki, N. }\end{array}$ & $\begin{array}{l}\text { Female entrepreneurship challenges in } \\
\text { Iran's co-operative sector. }\end{array}$ & 2012 & $\begin{array}{l}\text { Advanced Materials Re- } \\
\text { search Trans Tech Publi- } \\
\text { cations }\end{array}$ \\
\hline $\begin{array}{l}\text { Rezaei, J.; } \quad \text { Ortt, } \quad \text { R.; } \\
\text { Scholten, V. }\end{array}$ & $\begin{array}{l}\text { An improved fuzzy preference pro- } \\
\text { gramming to evaluate entrepreneur- } \\
\text { ship orientation. }\end{array}$ & 2013 & Apllied Soft Computing \\
\hline $\begin{array}{l}\text { Rostamzadeh, R.; Ismall, } \\
\text { K.; Noubar, H. B. K. }\end{array}$ & $\begin{array}{l}\text { An application of a hybrid MCDM } \\
\text { method for the evaluation of entrepre- } \\
\text { neurial intensity among the SMFs: A } \\
\text { case study. }\end{array}$ & 2014 & $\begin{array}{l}\text { The Scientific World } \\
\text { Journal }\end{array}$ \\
\hline $\begin{array}{l}\text { Schwartz, M.; Gothner, } \\
\text { M. }\end{array}$ & $\begin{array}{l}\text { A multidimensional evaluation of the } \\
\text { effectiveness of business incubators: } \\
\text { An application of the PROMETHEE } \\
\text { outranking method. }\end{array}$ & 2009 & $\begin{array}{l}\text { Environment and Plan- } \\
\text { ning C: Governamental } \\
\text { and Policy }\end{array}$ \\
\hline $\begin{array}{l}\text { Sha, K. H. C. K.; Hung, } \\
\text { C. Y.; Lin, B. S. P. }\end{array}$ & $\begin{array}{l}\text { Evaluating development strategies for } \\
\text { Tawan's iB3G industry: a fuzzy MCDM } \\
\text { approach. }\end{array}$ & 2004 & $\begin{array}{c}\text { Engineering Management } \\
\text { Conference, 2004. Pro- } \\
\text { ceedings, 2004. IEEE } \\
\text { International. }\end{array}$ \\
\hline $\begin{array}{l}\text { Tsai, W. H.; Lee, P. L.; } \\
\text { Shen, Y. S.; Hwang, E. T. }\end{array}$ & $\begin{array}{l}\text { A combined evaluation model for en- } \\
\text { couraging entrepreneurship policies. }\end{array}$ & 2014 & $\begin{array}{l}\text { Annals of Operations } \\
\text { Research }\end{array}$ \\
\hline $\begin{array}{l}\text { Tsai, Wen-Hsien; Kuo, } \\
\text { Hsiao-Chiao. }\end{array}$ & $\begin{array}{l}\text { Entrepreneurship policy evaluation and } \\
\text { decision analysis for SMEs. }\end{array}$ & 2011 & $\begin{array}{l}\text { Expert Systems with Ap- } \\
\text { plications }\end{array}$ \\
\hline
\end{tabular}

Fonte: Dados da Scopus e ISI Web of Science. Elaborado pelos autores 


\subsection{Ciclos de Produção - Quantitativo de artigos por ano}

De acordo com a Figura 1, os artigos referentes à aplicação da análise multicritério ao tema empreendedorismo teve início no ano de 2004, a produção de artigos manteve-se pequena e constante até o ano de 2011.

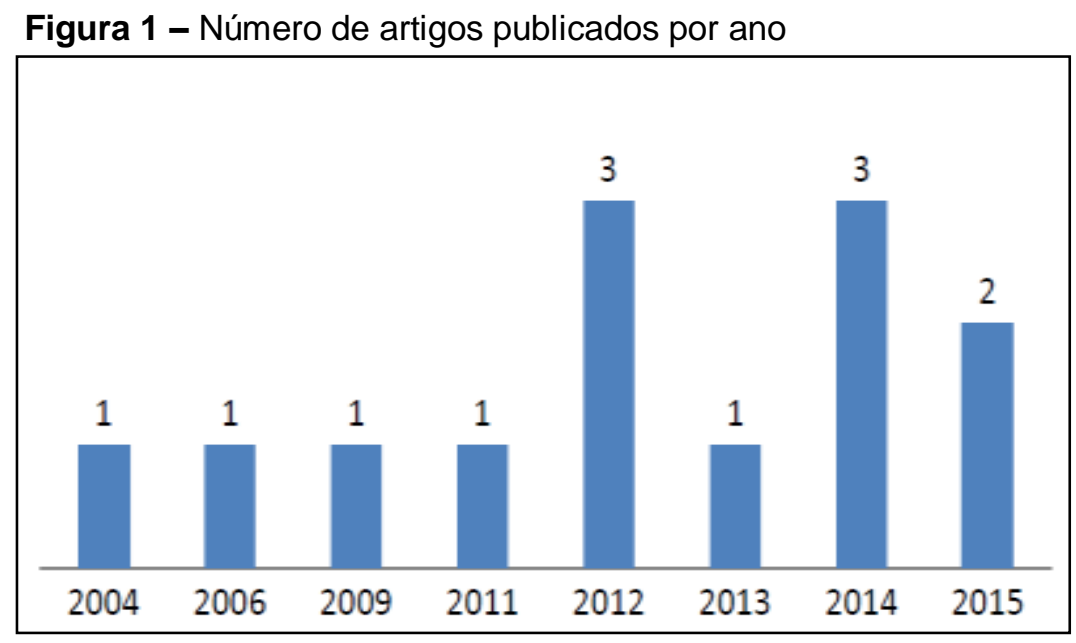

Fonte: Dados da Scopus e ISI Web of Science. Elaborado pelos autores

Os anos de 2012 e 2014 foram os anos com um maior número de publicações com três artigos.

\subsection{Análise das palavras-chave}

As palavras-chaves, os resumos e os títulos dos artigos resultantes da pesquisa podem ser observados na nuvem de palavras ilustrada na Figura 2.

A nuvem de palavras foi formada a partir do uso do software Wordle, o qual permite a visualização das palavras em tamanho proporcional à frequência em que as mesmas aparecem na pesquisa. 
Figura 2 - Nuvem de palavras-chave dos artigos da pesquisa

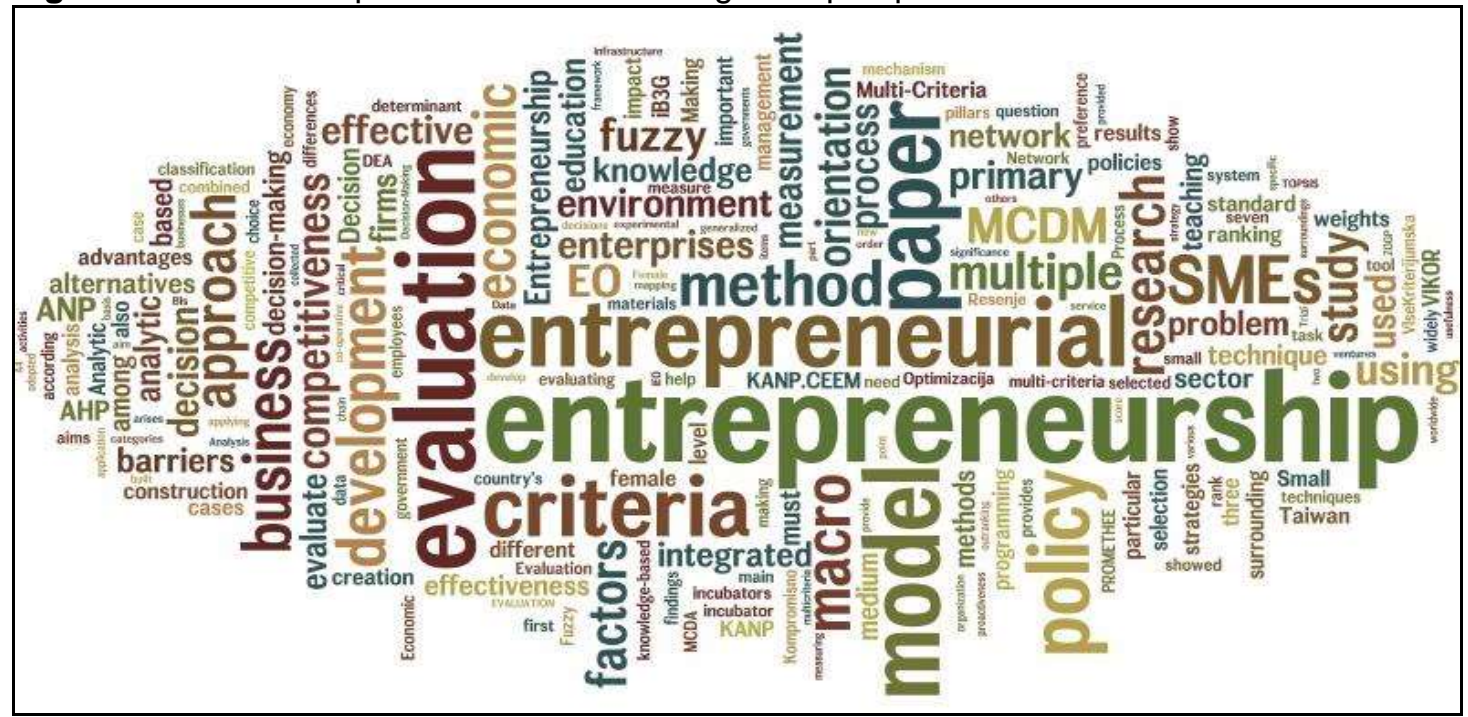

Fonte: Wordle (http://www.wordle.net/create)

De acordo com a nuvem, pode-se concluir que os artigos, em sua maioria, tratam de empreendedorismo, educação, avaliação, método, MCDM, MCDA, negócios, pesquisa, economia, critérios e desenvolvimento.

\subsection{Análise do país de origem}

Considerando o quantitativo de artigos por país resultantes da pesquisa, catorze países foram identificados: Alemanha, Austrália, Brasil, China, Estados Unidos, Hong Kong, Irã, Lituânia, Malásia, Nova Zelândia, Países Baixos, Portugal, Reino Unido e Taiwan. Com exceção do Reino Unido e Irã que apresentaram três publicações, os demais, incluindo o Brasil, tiveram apenas um trabalho identificado. Conforme ilustrado na Figura 3. 
Figura 3-Gráfico de país de origem

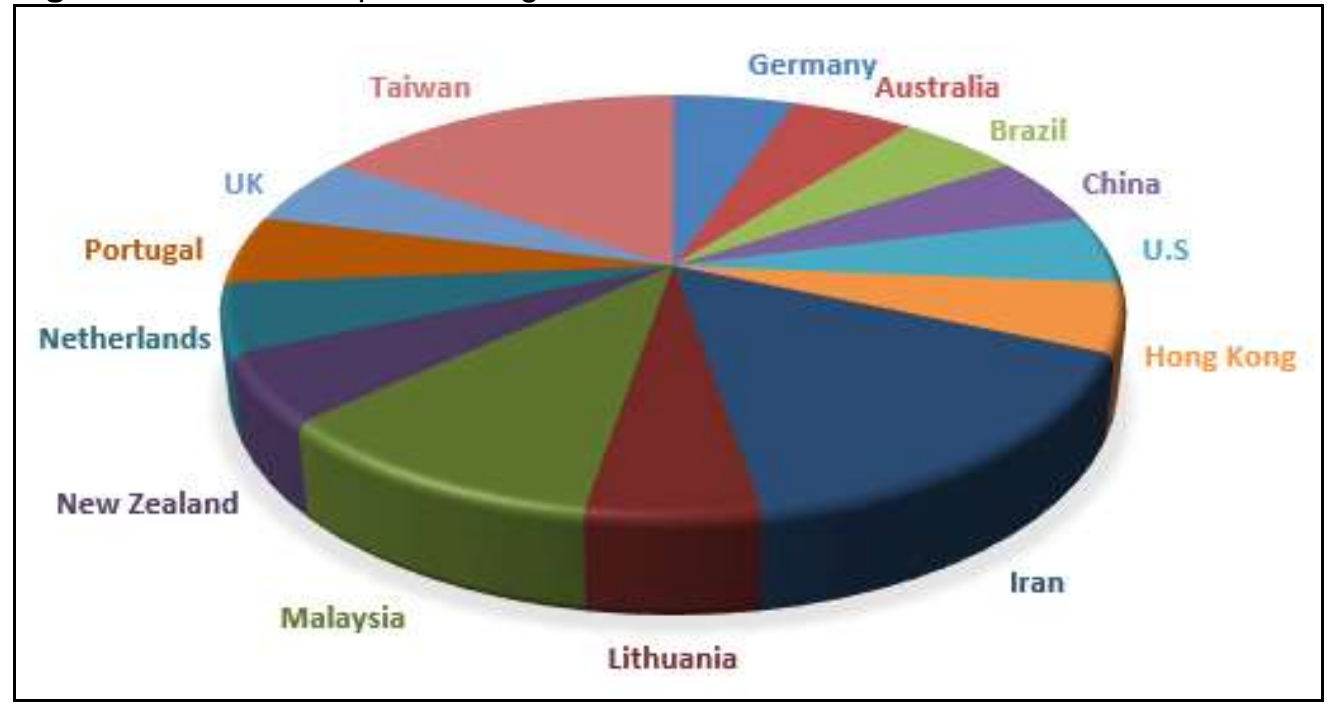

Fonte: Dados da Scopus e ISI Web of Science. Elaborado pelos autores

\subsection{Autores com maior número de publicações}

Da mesma forma que as instituições, os artigos apontados pela pesquisa são de diferentes autores, não havendo aparentemente uma grande referência na área, os autores identificados na pesquisa apresentam um artigo cada.

Figura 4 - Clusters principais com os autores do tema

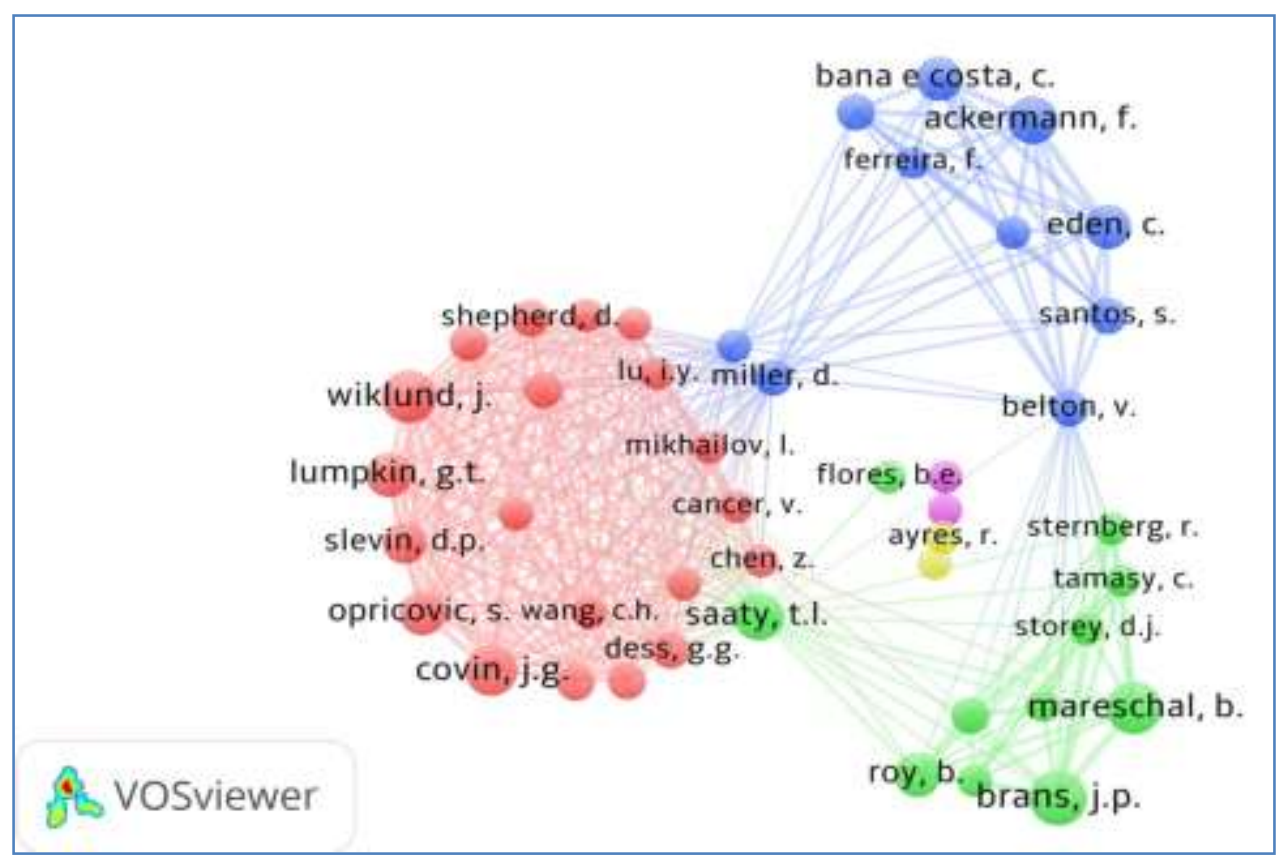

Fonte: VOSviewer. 
Entretanto, a partir dos 53 autores identificados, não fazendo distinção entre autor e co-autor, considerando as referências em cada artigo é possível alcançar 45 autores distribuídos em 5 clusters, que tiveram pelo menos 3 citações, conforme apresentado na Figura 4.

\section{5 Áreas do conhecimento em destaque}

As áreas de conhecimento que se destacam de acordo com a Figura 5 são engenharia (17\%), e gestão (13\%). As áreas de ciências da computação, negócios e contabilidade representam $10 \%$ cada.

Figura 5 - Artigos por área do conhecimento

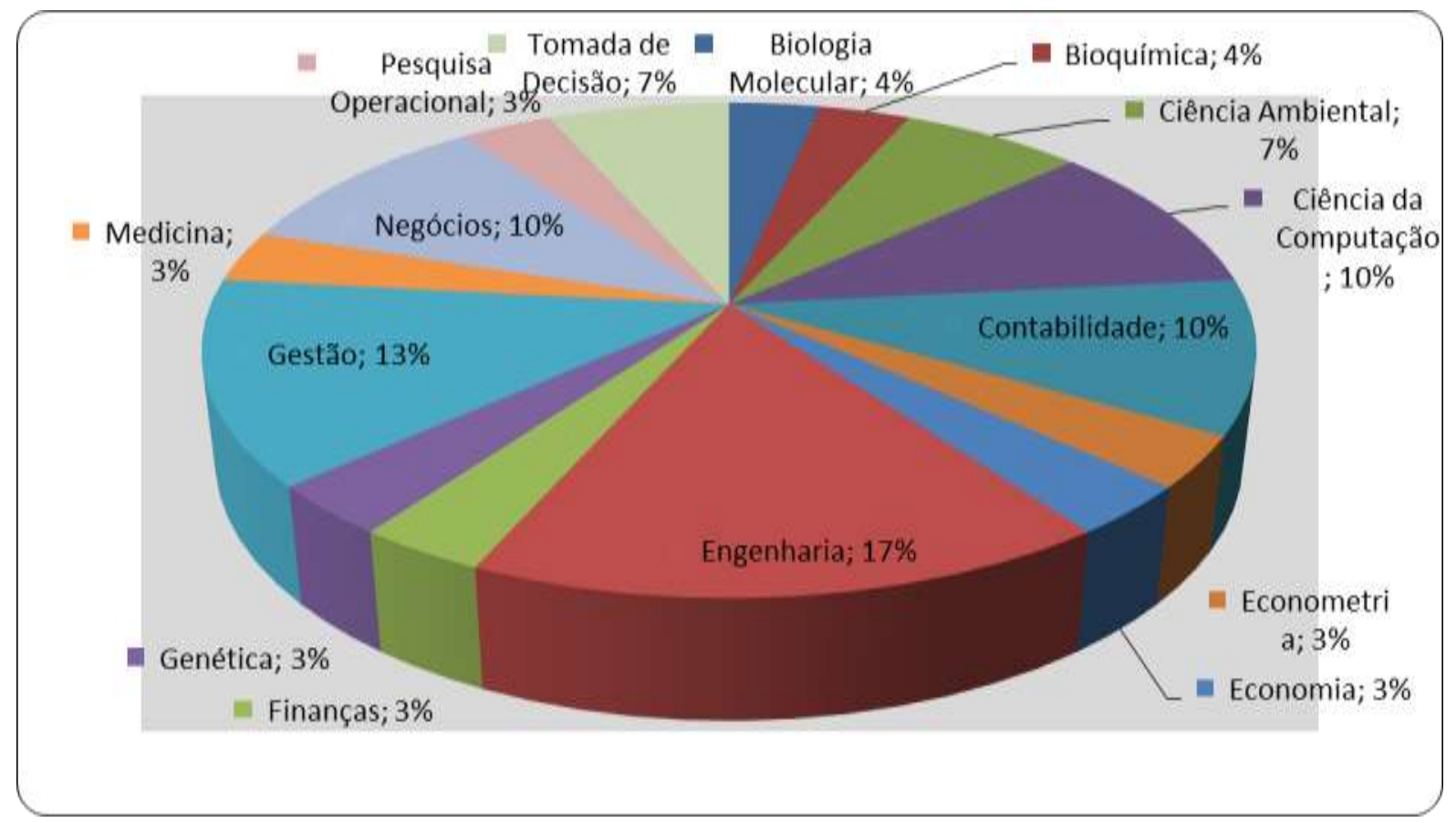

Fonte: Dados da Scopus e ISI Web of Science (2016). Elaborado pelos autores

\subsection{Periódicos identificados}

As publicações estão pulverizadas em 13 periódicos diversos. Não havendo nenhum que se destaque perante os demais conforme Quadro 3. 
Quadro 3 - Número de publicações por periódico

\begin{tabular}{|l|c|l|c|}
\hline \multicolumn{1}{|c|}{ Periódico } & Qt. & \multicolumn{1}{|c|}{ Periódico } & Qt. \\
\hline $\begin{array}{l}\text { IEEE International Engineering Manage- } \\
\text { ment Conference }\end{array}$ & 1 & Expert Systems with Applications & 1 \\
\hline Advanced Materials Research & 1 & Journal of Business Research & 1 \\
\hline Annals of Operations Research & 1 & Journal of Management Development & 1 \\
\hline Applied Mechanics and Materials & 1 & Pesquisa Operacional & 1 \\
\hline Applied Soft Computing Journal & 1 & $\begin{array}{l}\text { Proceedings - International Conference } \\
\text { on Machine Learning and Cybernetics }\end{array}$ & 1 \\
\hline Engineering Economics & 1 & Scientific World Journal & 1 \\
\hline $\begin{array}{l}\text { Environment and Planning C: Government } \\
\text { and Policy }\end{array}$ & 1 & & \\
\hline
\end{tabular}

Fonte: Dados da Scopus e ISI Web of Science. Elaborado pelos autores

\section{CONCLUSÃO}

Percebe-se, ao fazer uma busca nas bases ISI Web of Science e Scopus, que o empreendedorismo desempenha um papel cada vez mais importante no desenvolvimento econômico baseado no conhecimento.

A análise multicritério tem sido cada vez mais utilizada como uma ferramenta para trazer um melhor entendimento sobre o empreendedorismo, devido a subjetividade nas respostas, por exemplo, de pesquisas. Com a revisão bibliográfica, percebeu-se que o uso da MCDM pelas organizações está aumentando.

Como contribuição, a partir da bibliometria realizada neste trabalho, um núcleo de partida para a pesquisa de quais métodos MCDM tem sido utilizado para pesquisas voltadas ao empreendedorismo foi gerado, abrangendo artigos distribuídos por diferentes anos da produção científica do termo pesquisado, a fim de obter uma cobertura mais consistente e ampla.

A partir das palavras-chave, resumos e títulos encontrados na amostra, observa-se que uma grande quantidade de palavras contribui para a seleção e identificação dos trabalhos, isso pode ser observado na Figura 2, que apresenta palavras como empreendedorismo, educação, avaliação, método, MCDM, MCDA, negócios, pesquisa, economia, critérios e desenvolvimento, dentre outras.

Os resultados obtidos possibilitam inferir que há indícios de um número ainda incipiente de publicações no tema "Empreendedorismo e multicritério" no Brasil, somente um artigo. 
Dessa forma, o presente artigo atendeu ao objetivo proposto de apresentar um estudo bibliométrico compilando quais anos, autores, periódicos, instituições, áreas e países têm obtido destaque no que tange ao assunto multicritério e empreendedorismo, segundo as informações extraídas nas bases Scopus e ISI Web of Science.

Como sugestões para trabalhos futuros, um estudo semelhante incluindo outras bases pode fornecer outras informações e ampliar o conhecimento sobre a utilização de ferramentas para prospectar os cenários; além de um estudo baseado em análise de redes sociais (SNA) com utilização do software VOSviewer para ampliar as informações de relacionamento entre diferentes instituições e autores.

Como limitação do estudo percebe-se que não foi possível obter uma classificação geral em relação a outras bases, visto que a bibliometria considerou apenas duas bases de dados (Scopus e ISI Web of Science).

\section{REFERÊNCIAS}

BURACAS, A.; ZVIRBLIS, A.; JOKSIENE, I. Measurement of Entrepreneurship Macro Surrounding Advantages: Country's Economic Competitiveness Approach. Engineering Economics, v. 23, n. 1, p. 5-13, 2012. http://dx.doi.org/10.5755/j01.ee.23.1.1219

COSTA, H. G. Modelo para webibliomining: proposta e caso de aplicação. Revista FAE, Curitiba, v. 13, n. 1, jan./jun. 2010.

CHEN, Z.; LI; H.; KONG, S. C.; XU, Q. An analytic knowledge network process for construction entrepreneurship education. Journal of Management Development, v. 25, n. 1, p. 1127, 2006. http://dx.doi.org/10.1108/02621710610637936

CHOU, C.; SHEN, C.; HSIAO, H. Preliminary exploration of teaching excellence in technical institutes and colleges in Taiwan-presenting entrepreneurship education as an example.

Transportation, p. B915, 2010.

CRISTIAN-AURELIAN, P.; CRISTINA, S. P. Entrepreneurship Education and e-learning: A Perfect Match. Journal of Electrical and Electronics Engineering, v. 5, n. 1, p. 203, 2012.

DELUYI, A. H. M.; SOFIAN, S; HAMID, A. B. A.; KAZEMI, M. Screening Efficiency and Entrepreneurship Potential in Automotive Suppliers Using DEA. Applied Mechanics \& Materials, n. 554, 2014. http://dx.doi.org/10.4028/www.scientific.net/AMM.554.510

DUVAL-COUETIL, N., PILCHER, J., WEILERSTEIN, P., \& GOTCH, C. Undergraduate Involvement in Intellectual Property Protection at Universities: Views from Technology Transfer Professionals. Development, v. 11, p. 12, 2014. 
EVANS, R. S.; PARKS, J.; NICHOLS, S. The Idea to Product@ Program: An Educational Model Uniting Emerging Technologies, Student Leadership and Societal Applications. International Journal of Engineering Education, v. 23, n. 1, p. 95-104, 2007.

FERREIRA, F. A.; MARQUES, C. S.; BENTO, P.; FERREIRA, J. J.; e JALALI, M. S. Operationalizing and measuring individual entrepreneurial orientation using cognitive mapping and MCDA techniques. Journal of Business Research, v. 68, n. 12, 2691-2702, 2015. http://dx.doi.org/10.1016/j.jbusres.2015.04.002

GALLOWAY, L.; ANDERSON, M.; BROWN, W. Are engineers becoming more enterprising? A study of the potentials of entrepreneurship education. International Journal of Continuing Engineering Education and Life Long Learning, v. 16, n. 5, p. 355-365, 2006. http://dx.doi.org/10.1504/IJCEELL.2006.010958

GOMES, L. F. A. M.; MACHADO, M. A. S.; RANGEL, L. A. D. The multiple choice problem with interactions between criteria. Pesquisa Operacional, v. 35, n. 3, p. 523-537, 2015. http://dx.doi.org/10.1590/0101-7438.2015.035.03.0523

GOMEZELJ, D.; KUŠCE, I. The influence of personal and environmental factors on entrepreneurs' performance. Kybernetes, v. 42, n. 6, p. 906-927, 2013.

http://dx.doi.org/10.1108/K-08-2012-0024

GONÇALVES FILHO, C. et al. Strategy, market orientation and entrepreneurship: proposition and test of rival business performance prediction models. International Journal of Entrepreneurship and Innovation Management, v. 15, n. 4, p. 275-291, 2012.

http://dx.doi.org/10.1504/IJEIM.2012.048082

GUGLIELMETTI, F. R.; MARINS, F. A. S.; SALOMON, V. A. P. Comparação teórica entre métodos de auxílio à tomada de decisão por múltiplos critérios. In: SIMPÓSIO BRASILEIRO DE PESQUISA OPERACIONAL., 35., 2003. Anais... Natal-RN, 2003.

HAN, J.; YANG, Y.; ZHAO, Y. Evaluation of entrepreneurial environment based on fuzzy comprehensive evaluation method. In: MACHINE LEARNING AND CYBERNETICS (ICMLC), 2012 INTERNATIONAL CONFERENCE ON. IEEE, Anais... 2012. p. 305-309. http://dx.doi.org/10.1109/ICMLC.2012.6358930

KHANDUJA, D.; KAUSHIK, P. Exploring education driven entrepreneurship in engineering graduates in India. International Journal of Continuing Engineering Education and Life Long Learning, v. 19, n. 2-3, p. 256-270, 2009.

http://dx.doi.org/10.1504//JCEELL.2009.025032

KARIMI, S.; CHIZARI, M.; BIEMANS, H. J.; MULDER, M. Entrepreneurship education in Iranian higher education: The current state and challenges. European Journal of Scientific Research, v. 48, n. 1, p. 35-50, 2010.

JAMALI, R.; FIROUZI JAHANTIGH, F.; SAZVAR, A.; MALEKI, N. Female Entrepreneurship Challenges in Iran's Co-Operative Sector. Advanced Materials Research. Trans Tech Publications, 2012. p. 2226-2229. http://dx.doi.org/10.4028/www.scientific.net/AMR.433$\underline{440.2226}$

PAPAYANNAKIS, L., KASTELLI, I., DAMIGOS, D. MAVROTAS, G. Fostering entrepreneurship education in engineering curricula in Greece. Experience and challenges for a Technical University. European Journal of Engineering Education, v. 33, n. 2, p. 199-210, 2008. http://dx.doi.org/10.1080/03043790801980086 
PARDEDE, E.; LYONS, J. Redesigning the assessment of an entrepreneurship course in an information technology degree program: Embedding assessment for learning practic-

es. Education, IEEE Transactions on, v. 55, n. 4, p. 566-572, 2012.

http://dx.doi.org/10.1109/TE.2012.2199757

QIAN, H.; HAYNES, K. E. Beyond innovation: the Small Business Innovation Research program as entrepreneurship policy. The Journal of Technology Transfer, v. 39, n. 4, p. 524543, 2014. http://dx.doi.org/10.1007/s10961-013-9323-x

REZAEI, J.; ORTT, R.; SCHOLTEN, V. An improved fuzzy preference programming to evaluate entrepreneurship orientation. Applied Soft Computing, v. 13, n. 5, p. 2749-2758, 2013. http://dx.doi.org/10.1016/j.asoc.2012.11.012

ROSTAMZADEH, R.; ISMAIL, K.; BODAGHI, K. N. H. An application of a hybrid MCDM method for the evaluation of entrepreneurial intensity among the SMEs: a case study. The Scientific World Journal, v. 2014, 2014. http://dx.doi.org/10.1155/2014/703650

RUDA, W.; MARTIN, T. A.; DANKO, B. Target group-specific design of student entrepreneurship support-A German example focusing on start-up motives and barriers. Acta Polytechnica Hungarica, v. 6, n. 3, p. 5-22, 2009.

RUDA, W.; MARTIN, T. A.; DANKO, B. Comparing Start-up Propensities and Entrepreneurship Characteristics of Students in Russia and Germany. Acta Polytechnica Hungarica, v. 9, n. 3, 2012.

SAEE, J. A critical evaluation of Australian entrepreneurship education and training. In: Proceedings of the Internationalizing Entrepreneurship Education and Training Conference. 1996.

SCHWARTZ, M.; GÖTHNER, M. A multidimensional evaluation of the effectiveness of business incubators: an application of the PROMETHEE outranking method. Environment and Planning C: Government and Policy, v. 27, n. 6, p. 1072-1087, 2009.

SHA, K. H. C. K.; HUNG, C. Y.; LIN, B. S. P. Evaluating development strategies for Taiwan's iB3G industry: a fuzzy MCDM approach. In: Engineering Management Conference, 2004. Proceedings. 2004 IEEE International. IEEE, 2004. p. 258-262.

http://dx.doi.org/10.1109/IEMC.2004.1407116

SILVA, G. B; BARROS, M. D; COSTA, H. G. Entrepreneurship Education: A brief literature review. I Encontro Interestadual de Engenharia de Produção. São João da Barra-RJ, 2015a.

SILVA, G. B;; COSTA, H. G.; BARROS, M. D. Entrepreneurship in Engineering Education: A Literature Review. The International Journal of Engineering Education, v. 31, n. 6(A), p. 1701-1710, 2015b.

SILVA, A. C. C. J.; FURTADO, J. H.; ZANINI, R. R. Evolução do empreendedorismo no Brasil baseada nos indicadores do global entrepreneurship monitor (GEM). Revista Produção Online, v. 15, n. 2, p. 758-780, 2015. 10.14488/1676-1901.v15i2.1940

STAMBOULIS, Y.; BARLAS, A. Entrepreneurship education impact on student attitudes. The International Journal of Management Education, v. 12, n. 3, p. 365-373, 2014. http://dx.doi.org/10.1016/i.ijme.2014.07.001 
TIAN, Y.; WEN, C.; HONG, S. Global scientific production on GIS research by bibliometric analysis from 1997 to 2006. The Journal of Informetrics, v. 2, n. 1, p. 65-74, 2008. http://dx.doi.org/10.1016/j.joi.2007.10.001

TSAI, W.; KUO, H. Entrepreneurship policy evaluation and decision analysis for SMEs. Expert Systems with Applications, v. 38, n. 7, p. 8343-8351,

2011.http://dx.doi.org/10.1016/j.eswa.2011.01.022

TSAI, W. H.; LEE, P. L.; SHEN, Y. S.; HWANG, E. T. A combined evaluation model for encouraging entrepreneurship policies. Annals of Operations Research, v. 221, n.1, p. 449468, 2014. http://dx.doi.org/10.1007/s10479-011-1029-6

VALLE, L. A.; GUTIERREZ, R. H.; COSTA, H. G. Estudo bibliométrico da produção científica em gestão de processos na saúde no Brasil. In: ENCONTRO NACIONAL DE ENGENHARIA DE PRODUÇÃO, 33., Anais... Salvador-BA, 2013.

VAN RAAN, A. F. J. Comparison of the Hirsch-index with standard bibliometric indicators and with peer judgment for 147 chemistry research groups. Scientometrics, v. 67, n. 3, p. 491502, 2006. http://dx.doi.org/10.1007/s11192-006-0066-4

ZAPPE, S.; HOCHSTEDT, K.; KISENWETHER, E.; SHARTRAND, A. Teaching to innovate: Beliefs and perceptions of instructors who teach entrepreneurship to engineering students. The International journal of engineering education, v. 29, n. 1, p. 45-62, 2013.

ZHANG, G.; XIE, S.; HO, Y. S. A bibliometric analysis of world volatile organic compounds research trends. Scientometrics, v. 83, n. 2, p.477-492, 2009.

http://dx.doi.org/10.1007/s11192-009-0065-3

ZYOUD, S. E. H.; AL-JABI, S. W.; SWEILEH, W. M.; AWANG, R. A bibliometric analysis of toxicology research productivity in Middle Eastern Arab countries during a 10-year period (2003-2012). Health research policy and systems/Bio Med Central, v.12, p.4, 2014.

YANG, D.; ZHAO, X. Study on Evolutionary Path of University Students' Entrepreneurship Training. Mathematical Problems in Engineering, 2014.

http://dx.doi.org/10.1155/2014/535137

WILLERDING, I. A. V.; PRADO, M. L.; LAPOLLI, E. M. A trilogy of entrepreneurship: performance, capacity and competence as a factor of success for micro and small enterprises. IEEE Latin America Transactions, v. 10, n. 5, p. 2017-2024, 2012. http://dx.doi.org/10.1109/TLA.2012.6362343

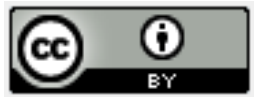

Artigo recebido em 03/03/2017 e aceito para publicação em 18/04/2017

DOI: http://dx.doi.org/10.14488/1676-1901.v17i3.2764 